\title{
Christel Coton, Officiers. Des classes en lutte sous
} l'uniforme

Marseille, Agone, coll. «L'Ordre des choses », 2017

\section{Mathias Thura}

\section{OpenEdition}

\section{Journals}

\section{Édition électronique}

URL : http://journals.openedition.org/travailemploi/8035

DOI : 10.4000/travailemploi.8035

ISSN : 1775-416X

Éditeur

DARES - Ministère du Travail

\section{Édition imprimée}

Date de publication : 1 janvier 2018

Pagination : $123-126$

ISSN : 0224-4365

\section{Référence électronique}

Mathias Thura, «Christel Coton, Officiers. Des classes en lutte sous l'uniforme », Travail et Emploi [En ligne], 153 | janvier-mars 2018, mis en ligne le 11 juillet 2019, consulté le 25 septembre 2020. URL : http://journals.openedition.org/travailemploi/8035; DOI : https://doi.org/10.4000/travailemploi.8035 


\section{Notes DE LECTURE}

\section{Officiers. Des classes en lutte sous l'uniforme}

\section{Christel Coton}

Marseille, Agone, coll. «L'Ordre des choses », 2017, 288 p.

\section{Lu par Mathias Thura*}

Dans cet ouvrage, Christel Coton s'attaque au «mythe méritocratique » au fondement du discours officiel porté sur les carrières au sein des armées. Si l'institution militaire aime à se présenter comme une authentique institution de la seconde chance (p. 17 et suivantes) - offrant aux plus méritants un véritable droit à la carrière et la possibilité de se hisser jusqu' au grade d'officier et d'accéder ainsi à l'épaulette -, l'auteure donne à voir une tout autre réalité. Partant des différences de trajectoires sociales, scolaires et professionnelles entre les officiers « promus par le rang » et ceux passés par l'École spéciale militaire de Saint-Cyr Coëtquidan (ESM) après un parcours en classes préparatoires, C. Coton analyse les mécanismes de (pré)sélection et de promotion qui président à l'identification et à la reproduction d'une élite au sein du groupe des officiers. Elle montre comment les membres de cette élite accumulent les marqueurs de prestige institutionnel et concentrent in fine les chances d'être appelés à occuper les postes à responsabilités grâce auxquels ils peuvent se faire remarquer et ainsi « briller sous l'épaulette ${ }^{1} »$. Proposant une sociologie des élites militaires dans la lignée des travaux inspirés par Pierre Bourdieu, l'ouvrage constitue une remarquable contribution à l' analyse des conditions sociales du charisme parmi les officiers, et plus généralement des conditions sociales de la reconnaissance du talent et du brio professionnel.

Associant entretiens, observations directes et questionnaires, l'enquête s'appuie sur deux terrains complémentaires : un premier réalisé au sein d'un régiment opérationnel et un second conduit à l'École d'état-major de l'armée de Terre. Ce second terrain a été l'occasion d'une observation en immersion parmi une promotion d'officiers qui, après un premier temps de commandement, réalisaient la formation leur permettant d'amorcer la deuxième partie de leurs carrières. C. Coton observe un moment où ces officiers sont mis directement en concurrence et mis à l'épreuve en vue d'être classés. Ce terrain s'avère ainsi particulièrement propice à la saisie de l'actualisation des petites (et grandes) différences qui contribuent à la rapide différenciation de carrières et qui sont pourtant ordinairement occultées par l'illusion d'égalité de positions évoquée plus haut.

\footnotetext{
* Laboratoire Sociétés, acteurs, gouvernement en Europe (Sage), université de Strasbourg.

1. Coton C. (2012), « Briller sous l'épaulette. Capital culturel et capital combattant dans le corps des officiers de l'armée de Terre », Actes de la recherche en sciences sociales, ${ }^{\circ}$ 191-192, pp. 14-17.
} 
L'ouvrage est composé de six chapitres. Le premier présente le cadre organisationnel et les règles qui régissent le fonctionnement de la promotion des militaires. Les différentes filières de recrutement des officiers y sont présentées, permettant au lecteur de se faire une idée de l'hétérogénéité relative des situations subsumées sous l'uniforme. Entre, d'une part, les jeunes cyrards, directement recrutés en tant qu'officiers à l'issue d'un passage en classes préparatoires et d'une scolarité de trois ans à l'ESM, les officiers sur titre (recrutés à bac +5 ), les officiers sous contrats (recrutés à bac +3 , contractuels et aux perspectives de promotion restreintes) et, d'autre part, les dolos et les oиаоиаs, recrutés parmi les sous-officiers à différents moments de leur carrière et passés par l'École militaire inter-armée (EMIA), elle aussi basée à Saint-Cyr, le corps des officiers se révèle parcouru de lignes de fractures. L'auteure analyse cette hétérogénéité et en révèle les structures en articulant les différences statutaires avec les origines sociales et les différences de trajectoires scolaires.

Les chapitres deux, trois et quatre examinent successivement les mécanismes de la domination parmi les officiers : l'horizon d'évaluation des carrières, les ressorts de la distinction entre officiers et les conditions du charisme guerrier. L'auteure s'intéresse d'abord à ce qu'elle nomme "l'étalon combattant », c'est-à-dire aux expériences de commandement en opération comme modalité cardinale de mesure de la légitimité professionnelle chez les officiers. Ces expériences constituent des ressources valorisées et valorisables pour se positionner dans la course à la promotion. Ce que montre C. Coton, c'est d'abord l'inégale probabilité d'accéder à cette ressource en fonction des armes et des spécialités. Les officiers issus des armes dites de «mêlée » ont toutes les chances de détenir ce marqueur de prestige alors que ceux issus des unités dites de « soutien » en sont tendanciellement écartés. De plus, l'organisation du stage à l'École d'état-major et son évaluation tendent à favoriser les détenteurs des compétences tactiques acquises dans les régiments de combat. Mais plus que cela, ce «capital combattant » s'avère à double tranchant : à la fois valorisable dans la mise en scène de soi entre officiers, il peut se retourner en stigmate, celui du «bourrin» manquant de finesse. Stigmate auquel les officiers issus du rang semblent plus exposés que leurs camarades saintcyriens. De plus, la mobilisation de dispositions plus strictement scolaires contribue aussi au processus d'émergence des « champions » parmi les stagiaires : prise de parole en groupe, esprit d' analyse et de synthèse, ascèse scolaire. Ainsi, si les combattants sont bien « du côté de la vérité des pratiques militaires, [et] surreprésentés dans les fonctions de haut commandement » (p. 153), le fait d'avoir un passé opérationnel prestigieux ne suffit pas à faire «le charisme guerrier». C'est la rencontre entre la classe sociale et le passé opérationnel qui contribue à la production de la domination d'une élite sur le reste des officiers. Une domination qui s'exerce autant par la maîtrise des compétences professionnelles attendues des officiers que par la manifestation d'une forme spécifique de manières d'être via l'usage de la voix, la mise en scène de soi et la mise en récit de ses expériences opérationnelles, la manifestation de la certitude de soi par l'art de la prise de décision rapide et ferme, la conduite de la conversation, le contrôle de soi, le rapport de proximité à la culture légitime, etc. À rebours du postulat d'égalité, 
C. Coton montre comment les officiers se classent perpétuellement entre eux à l'aune de ces critères qui traduisent des différences de positions sociales dans le langage de l'institution, en plus d'être régulièrement classés par l'institution elle-même par le biais d'évaluations et d'examens ponctuant leur carrière à plusieurs reprises.

Le chapitre cinq s'intéresse quant à lui aux « antichambres de la consécration », les classes préparatoires militaires, où s'acquièrent des dispositions à l'incarnation du charisme guerrier ainsi que les premiers marqueurs de l'excellence institutionnelle. En remontant le fil des trajectoires et en restituant ces dernières dans les contextes qui les ont produits (famille, école, etc.), le poids des socialisations antérieures apparaît particulièrement prégnant dans la constitution de ce qui est souvent considéré au sein des armées comme la stricte expression d'un don pour le commandement, justement caractéristique de l'élite parmi les officiers. L'exploration des multiples mécanismes d'élection sociale de cette élite se fait plus complète : ces officiers sont à la fois prédisposés - par la fréquentation d'instances socialisatrices particulières : le scoutisme, les classes préparatoires militaires, puis par le passage par l'ESM - à manifester les conduites et les tournures d'esprit consacrées dans l'institution, favorisés par les règles d'encadrement des carrières et plus facilement nommés à des postes de commandement combattant dès le début de leur carrière. Ils sont rapidement amenés à former, hors incident de parcours, une frange spécifique de la population des officiers, qui accumule très tôt les marqueurs de l'excellence militaire.

Le sixième et dernier chapitre clôt l'analyse en proposant une interprétation de la culture militaire comme l'expression d'une culture de classe militarisée. Les relations quotidiennes entre officiers se façonnent sur fond de réseaux de sociabilités distincts, liés au partage ou non de pratiques socialement situées, et plus particulièrement à « une sociabilité religieuse et bourgeoise [qui] contribue à alimenter les frontières [...] saisies dans leur dimension militaire » (p. 236). L'auteure répond ainsi à la question qu'elle s'est donnée dès l'introduction : retrouver l'expression des classes sociales sous l'uniforme. Les mécanismes présentés tout au long de l'ouvrage sont ainsi autant de pièces qui permettent de comprendre comment les différences d'origine sociale et de trajectoire scolaire sont retraduites sous formes de clivages structurant les relations entre collègues. Cela permet à l'auteure de conclure sur les efforts (vains ?) et la bonne volonté culturelle (mise en échec ?) de ceux qui sont dès le départ exclus des cercles et des instances de la reproduction de cette élite.

La lecture de cet ouvrage ouvre de nombreuses perspectives, tant du côté de la sociologie de l'institution militaire que du côté de la sociologie des élites, ainsi que de la sociologie des groupes professionnels, bien que l'auteure fasse l'impasse sur cette littérature. Il s'agit d'une importante contribution à l'analyse des dispositifs de carrière et des mécanismes de socialisation des élites militaires, complétant le travail entamé par Claude Weber à Saint-Cyr Coëtquidan ${ }^{2}$. Cette enquête apporte également une

2. Weber C. (2012), À genou les hommes, debout les officiers. La socialisation des Saint-Cyriens, Rennes, Presses universitaires de Rennes. 
pierre supplémentaire à la connaissance des mécanismes de sélection et de promotion au sein de la haute fonction publique. Elle met à la fois en évidence l'importance persistante des capitaux scolaires tout au long de la promotion des officiers, mais aussi un vaste ensemble de déterminants sociaux à l'œuvre derrière la réglementation des carrières. Elle confirme de la sorte ce qui a largement été documenté du côté de l'institution scolaire - la reproduction des hiérarchies sociales à travers la sélection scolaire -, mais prolonge et transpose le résultat à la sélection et à la promotion professionnelle au sein d'un univers bureaucratique. C. Coton ne se contente pas de mettre en lumière les effets systémiques de cette sélection sociale, elle montre comment ces chances inégales sont aussi le produit d'un processus circonstancié et continu qui se joue et se rejoue dans les interactions ordinaires qui trament les sociabilités entre officiers. Enfin, l'ouvrage montre bien comment les modalités d'évaluation de la compétence professionnelle traduisent dans une formulation technique (ici la maîtrise de la tactique, l'art du commandement, l'esprit de synthèse, etc.) la manifestation de dispositions sociales cultivées au sein d'instances de socialisation particulières (qui dépassent l'institution militaire à proprement parler). De la sorte, C. Coton apporte la démonstration convaincante que les cultures professionnelles - ici au sein d'une fonction publique particulière - peuvent aussi être appréhendées et pensées comme l'expression de cultures de classes. 